\title{
RESEARCH
}

Open Access

\section{Real-time assessment of right and left ventricular volumes and function in children using high spatiotemporal resolution spiral bSSFP with compressed sensing}

Jennifer A. Steeden', Grzegorz T. Kowalik', Oliver Tann², Marina Hughes², Kristian H. Mortensen² and Vivek Muthurangu ${ }^{1 *}$

\begin{abstract}
Background: Real-time cardiovascular magnetic resonance (CMR) assessment of ventricular volumes and function enables data acquisition during free-breathing. The requirement for high spatiotemporal resolution in children necessitates the use of highly accelerated imaging techniques.

Methods: A novel real-time balanced steady state free precession (bSSFP) spiral sequence reconstructed using Compressed Sensing (CS) was prospectively validated against the breath-hold clinical standard for assessment of ventricular volumes in 60 children with congenital heart disease. Qualitative image scoring, quantitative image quality, as well as evaluation of biventricular volumes was performed. Standard BH and real-time measures were compared using the paired t-test and agreement for volumetric measures were evaluated using Bland Altman analysis.
\end{abstract}

Results: Acquisition time for the entire short axis stack ( 13 slices) using the spiral real-time technique was $\sim 20 \mathrm{~s}$, compared to $\sim 348 \mathrm{~s}$ for the standard breath hold technique. Qualitative scores reflected more residual aliasing artefact $(p<0.001)$ and lower edge definition $(p<0.001)$ in spiral real-time images than standard breath hold images, with lower quantitative edge sharpness and estimates of image contrast $(p<0.001)$.

There was a small but statistically significant $(p<0.05)$ overestimation of left ventricular $(L V)$ end-systolic volume $(1.0 \pm 3.5 \mathrm{~mL})$, and underestimation of LV end-diastolic volume $(-1.7 \pm 4.6 \mathrm{~mL})$, LV stroke volume $(-2.6 \pm 4.8 \mathrm{~mL})$ and LV ejection fraction $(-1.5 \pm 3.0 \%)$ using the real-time technique. We also observed a small underestimation of right ventricular stroke volume $(-1.8 \pm 4.9 \mathrm{~mL})$ and ejection fraction $(-1.4 \pm 3.7 \%)$ using the real-time imaging technique. No difference in inter-observer or intra-observer variability were observed between the $\mathrm{BH}$ and real-time sequences.

Conclusions: Real-time bSSFP imaging using spiral trajectories combined with a compressed sensing reconstruction showed good agreement for quantification of biventricular metrics in children with heart disease, despite slightly lower image quality. This technique holds the potential for free breathing data acquisition, with significantly shorter scan times in children.

Keywords: Real-time, Left ventricular volumes, Right ventricular volumes, Spiral, Compressed sensing, Paediatric, Children, Congenital heart disease

\footnotetext{
* Correspondence: v.muthurangu@ucl.ac.uk

${ }^{1}$ UCL Centre for Cardiovascular Imaging, Institute of Cardiovascular Science,

University College London, 30 Guildford Street, London WC1N 1EH, UK

Full list of author information is available at the end of the article
}

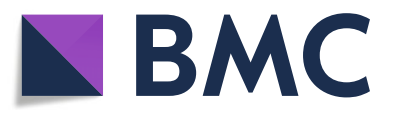

(c) The Author(s). 2018 Open Access This article is distributed under the terms of the Creative Commons Attribution 4.0 International License (http://creativecommons.org/licenses/by/4.0/), which permits unrestricted use, distribution, and reproduction in any medium, provided you give appropriate credit to the original author(s) and the source, provide a link to the Creative Commons license, and indicate if changes were made. The Creative Commons Public Domain Dedication waiver (http://creativecommons.org/publicdomain/zero/1.0/) applies to the data made available in this article, unless otherwise stated. 


\section{Background}

Evaluation of biventricular volumes and function is vital in the investigation of children with congenital heart disease. The clinical standard method of evaluating ventricular volumes is cardiovascular magnetic resonance (CMR) - specifically, multi-slice cardiac gated balanced steady state free precession (bSSFP) cine imaging [1]. This technique is now routinely used in children and has an important role to play in disease management [2]. Unfortunately, it does require multiple breath-holds $(\mathrm{BH})$ and this can be difficult in young children with heart disease. An alternative approach is real-time CMR in which each $k$-space is acquired in a single shot manner, rather than over several heart beats. The benefit of real-time CMR is that it can be acquired during free breathing and without cardiac gating. However, this comes at the cost of lower spatial and temporal resolution, which can affect accuracy [3].

One solution is to leverage accelerated imaging techniques (data undersampling or more efficient $k$-space filling) to improve resolution. Recently, a combination of undersampled radial $k$-space filling and compressed sensing (CS) has been shown to enable relatively high resolution real-time imaging $[4,5]$. Studies in adults have demonstrated good agreement between these radial real-time techniques and the breath-hold clinical standard for assessment of ventricular volumes [6-9]. However, imaging children requires much higher spatial and temporal resolution than available using conventional radial CS methods. Hence greater acceleration is needed to translate these techniques into the paediatric population. One possibility is to combine CS with more efficient spiral $k$-space filling. Spiral trajectories have previously been used to accelerate real-time phase contrast CMR with some success [10]. They can also be combined with golden angle spacing $[11,12]$ to enforce incoherent aliasing necessary for CS reconstruction.

In this study, we implemented a novel real-time bSSFP spiral sequence reconstructed using CS. The purpose of this study was to validate this real-time sequence against the $\mathrm{BH}$ clinical standard for assessment of biventricular volumes in children with heart disease.

\section{Methods}

\section{Study population}

Between December 2017 and February 2018, 60 consecutive children referred for CMR and consented for additional research scans were included into this study. The local committee of the UK National Research Ethics Service approved the study (06/Q0508/124), and written consent was obtained from all subjects/guardians. Patients were excluded from this study if: a) there was a requirement for general anesthetic (15 children excluded during the recruitment period), b) the subject had an irregular heart rate or difficulty performing breath-holds (11 children excluded), or c) the subject had single ventricular anatomy which was excluded to ensure that biventricular volumes could be evaluated in all patients (4 children excluded).

\section{Imaging protocol}

All imaging was performed on a 1.5 Tesla CMR scanner (Avanto, Siemens Healthineers, Erlangen, Germany) with maximum gradient amplitude of $40 \mathrm{mT} / \mathrm{m}$ and a maximum slew rate of $180 \mathrm{~T} / \mathrm{m} / \mathrm{s}$. Signal detection was accomplished using two spine coils incorporated into the scan table and arranged in head-foot manner, and one anteriorly placed body-matrix coil (giving a total of 12 coil elements). A vector electrocardiographic system was used for cardiac gating.

\section{Standard volumetric assessment}

Standard cardiac-gated ventricular volume assessment was performed using a multi-slice retrospectively cardiac gated bSSFP Cartesian sequence. The temporal resolution was $29.5 \mathrm{~ms}$ and the in-plane spatial resolution was $1.5 \times 1.5 \mathrm{~mm}$ (full sequence parameters are shown in Table 1). The imaging plane was in the ventricular short axis (SAX), planned using right ventricular (RV) and left ventricular (LV) long axes and 4-chamber images. Sufficient contiguous slices were acquired in the short axis to ensure coverage of the whole ventricle $(\sim$ $13 \pm 2$ slices, range: 10 to 17 ). Each slice was acquired in a separate breath-hold, each lasting $\sim 5.5 \pm 1.1 \mathrm{~s}$ (range: 3.6 to $8.8 \mathrm{~s})$.

\section{Real-time volumetric assessment}

Real-time bSSFP imaging was performed using a 2D multi-slice uniform density spiral sequence. The spiral trajectories were designed using the method described by Hargreaves [13], assuming a field-of-view of $350 \mathrm{~mm}$, a spatial resolution of $\sim 1.7 \times 1.7 \mathrm{~mm}$ and 72 regularly spaced spiral interleaves for complete filling of $k$-space. A zeroth moment rewinder gradient was added at the end of the spiral readout to maintain bSSFP coherence (see Fig. 1 for pulse sequence diagram). In this study we did not use bipolar first moment rewinder gradients, due to the associated increase in TR [14]. Consecutive interleaves were rotated by the tiny golden angle ( $\mathrm{tGA}, \sim 47.26^{\circ}$ ) throughout data acquisition. This enforced incoherent aliasing required for the CS reconstruction, whilst being associated with lower eddy currents than traditional golden angle sampling patterns [15]. Each real-time frame was formed by combining 9 consecutive spiral interleaves (acceleration factor $=8$ ), resulting in a temporal resolution of $\sim 30 \mathrm{~ms}$. The full sequence parameters are shown in Table 1. 
Table 1 Sequence parameters

\begin{tabular}{|c|c|c|}
\hline & $\begin{array}{l}\text { Standard BH } \\
\text { technique }\end{array}$ & $\begin{array}{l}\text { Spiral real-time } \\
\text { technique }\end{array}$ \\
\hline Field of view (mm) & 350 & 350 \\
\hline $\begin{array}{l}\text { Rectangular field-of- } \\
\text { view (\%) }\end{array}$ & 75 & 100 \\
\hline Matrix size & $240 \times 180$ & $208 \times 208$ \\
\hline Number of slices & 13 (range: 10-17) & 13 (range: 10-17) \\
\hline Image resolution (mm) & $1.46 \times 1.46 \times 8$ & $1.68 \times 1.68 \times 8$ \\
\hline TE/TR (ms) & $1.16 / 2.32$ & $0.67 / 3.34$ \\
\hline Flip Angle (degree) & 67 & 67 \\
\hline $\begin{array}{l}\text { Pixel Bandwidth } \\
\text { (Hz/pixel) }\end{array}$ & 1225 & 1502 \\
\hline Cardiac gating & Retrospective & - \\
\hline $\begin{array}{l}\text { Temporal resolution } \\
\text { (ms) }\end{array}$ & 29.48 & 30.06 \\
\hline $\begin{array}{l}\text { Reconstructed cardiac } \\
\text { phases }\end{array}$ & 40 & 24 (range: 20-37) \\
\hline Trajectory & Cartesian & Spiral \\
\hline $\begin{array}{l}\text { Fully sampled spiral } \\
\text { interleaves }\end{array}$ & - & 72 \\
\hline GRAPPA & $\times 2$ & - \\
\hline Compressed Sensing & - & $\times 8$ \\
\hline $\begin{array}{l}\text { Breath-hold time per } \\
\text { slice (sec) }\end{array}$ & $3.6-8.8$ & - \\
\hline $\begin{array}{l}\text { Total acquisition time } \\
\text { for SAX (sec) }\end{array}$ & 350 (range: 239-573) & $\sim 20$ (range: 11 to 36 ) \\
\hline
\end{tabular}

$\mathrm{BH}$, breath hold; TE, echo time; TR, repetition time, GRAPPA, generalized autocalibrating partially parallel acquisition

Each slice was acquired over two R-R intervals. The first was used to reach the steady state and the second was used for acquisition of data. Synchronisation of acquisition of data to the start of the R-wave for each slice also ensured the real-time images were temporally aligned for measurement purposes. After acquisition of each slice, the imaging plane was moved to the next contiguous slice. The imaging plane and the number of slices was the same as the clinical standard $\mathrm{BH}$ sequence. All real-time image acquisition was performed during free breathing.

The CS reconstruction was performed on an external GPU equipped computer (Tesla K40c, NVIDIA Corportation, Santa Clara, California, USA) with on-line communication to the native reconstructor $[16,17]$. Our implementation of CS solved a set of non-linear equations (representing the imaging process) through minimization of the following cost function:

$$
\underset{\rho}{\operatorname{argmin}}\left\{\|E \rho-y\|_{2}^{2}+\lambda_{1}\left\|T V_{t} \rho\right\|_{1}\right\}
$$

The first term enforces data consistency - where $\rho$ is image data, $E$ is the encoding matrix (the multi-coil non-uniform Fourier transform (FT) operator) and $y$ is the acquired $k$-space data. The second term enforces sparse results through $\mathrm{L}_{1}$ norm regularization. A temporal finite difference operator $\left(T V_{t}\right)$, was used transform the image data into a sparser representation. This is similar to the previously described iterative Golden-angle Radial Sparse Parallel (GRASP) reconstruction [18]. The optimization was performed using a non-linear Conjugate Gradient algorithm, with 35 iterations. The regularization parameter $(\lambda)$ were selected empirically on the first 10 patients, to optimize artefact removal without temporal blurring and was set at 0.05 for all patients. As the data for each slice is independent, reconstruction started immediately after the last phase for each slice. Coil sensitivity information was calculated from the time-averaged data (from each slice) [19].

\section{Image processing and scoring}

Evaluation of ventricular volumes and qualitative image scoring was performed by three clinical CMR specialists
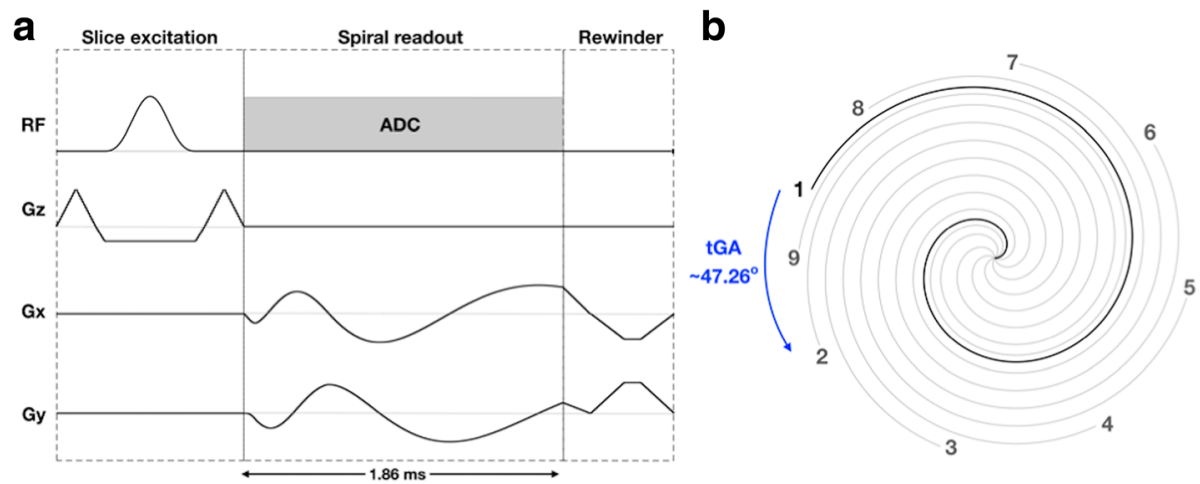

Fig. 1 Pulse sequence diagram and k-space trajectory design. a Pulse sequence diagram for the spiral real-time balanced steady state free precession (bSSFP) technique. TE $=0.67 \mathrm{~ms}, \mathrm{TR}=3.34 \mathrm{~ms}$. The refocusing nulls the zeroth moments of the gradient waveform only, $\mathbf{b}$ k-space trajectory, showing 9 consecutive TRs. The angle increment between successive spoke numbers is constant with an angle of $360^{\circ} /\left(6+\frac{(1+\sqrt{5})}{2}\right) \approx 47.26^{\circ}$ 
(OT - 12 years experience, $\mathrm{MH}-11$ years experience, and $\mathrm{KM}-5$ years experience). For both image quality scoring and assessment of LV and RV volumes, each clinician was the primary observer for 20 unique cases, of which 10 were re-evaluated to assess intra-observer variability. In addition, each observer assessed 10 cases from a different primary observer (5 cases from each of the other observers), to evaluate inter-observer variability. Thus, each observer scored and processed 40 cases. Overall 30 cases were used to evaluate intra-observer variability and the other 30 cases used to evaluate inter-observer variability.

\section{Image quality}

The mid-ventricular short-axis cine loops from each technique were scored on a 5-point Likert scale in three categories: sharpness of endocardial border $(1=$ nondiagnostic, $2=$ poor, 3 = adequate, $4=$ good, $5=$ excellent), temporal fidelity (or blurring) of wall motion $(1=$ nondiagnostic, $2=$ poor, 3 = adequate, $4=$ good, $5=$ excellent) and residual aliasing artefacts, which appear as spiral streaks across image $(1=$ non-diagnostic, $2=$ severe, $3=$ moderate, $4=$ mild, $5=$ minimal $)$. Each observer scored the patient data that they had previously segmented $(N=$ 40 cases each), at a separate session. All loops were presented in a random manner using a custom-built python application, with the observers blinded to diagnosis, patient number and type of sequence.

\section{Ventricular function}

Quantification of LV and RV volumes was performed in a similar manner for each technique using the OsiriX open source DICOM viewing platform (OsiriX v.9.0, OsiriX Foundation, Geneva, Switzerland) [20]. Firstly, the end-diastolic and end-systolic phases were identified for each ventricle through visual inspection of the midventricular cine. The endocardial borders of all slices at end systole and diastole were then traced manually (including papillary muscles and trabeculation in the myocardial mass). This allowed calculation of end-diastolic volume (EDV) and end-systolic volume (ESV). Stroke volume (SV) was obtained by subtracting ESV from EDV and ejection fraction $(E F)=S V / E D V \times 100$. In addition, LV epicardial borders were traced in end-diastole and combined with endocardial borders to obtain LV mass. Observers were presented with each anonymized volume (including repeated volumes for intra-observer variability) in a random order, blinded to diagnosis, patient number and type of sequence.

\section{Quantitative image assessment}

Calculation of signal-to noise ratio (SNR) and contrastto-noise ratio (CNR) in images reconstructed using CS is nontrivial due to the uneven distribution of noise.
Therefore, we calculated blood pool-to-myocardial signal intensity ratio [3] as a quantitative measure of image contrast, which is important when segmenting data. In all patients, the average blood pool and myocardial signal intensity values were measured in the end diastolic volume (from the LV endocardial and LV epicardial borders drawn for volumetric calculation, across the entire volume). The image contrast equalled blood pool signal intensity divided by myocardial signal intensity.

Edge sharpness of the mid-ventricular short-axis slice was evaluated by measuring the maximum gradient of the normalized pixel intensities across the border of the septum, as previously described [21]. To reduce noise, which results in artificially high gradients (representing sharp edges), the pixel intensities were fit to a tenth order polynomial, before differentiation. Edge sharpness was calculated in six positions across the septum, for all cardiac phases, and the average value was used for comparison.

\section{Statistics}

Statistical analyses were performed by using STATA software (STATA SE, v.14.2, Stata Corporation, College Station, Texas, USA). All results are expressed as the mean \pm standard deviation. Mean ventricular volumes, function and mass measured using the standard $\mathrm{BH}$ and spiral real-time techniques were compared using a paired t-test. For assessment of agreement of ventricular volumes and function, the standard BH data was used as the clinical standard for Bland-Altman analysis. Association of the percentage differences between the real-time and $\mathrm{BH}$ data against both heart rate and body surface area were tested using Spearman's rank correlation coefficient. Inter and intra-observer variability was assessed using the coefficient of variation $(\mathrm{CoV})$ calculated using the logarithmic method described by Bland and Altman [22]. In addition, inter-observer and intra-observer variability were also assessed using one-way intraclass correlation coefficient (ICC). Both $\mathrm{CoV}$ and ICC were displayed with their 95\% confidence intervals. Qualitative and quantitative image scores were also compared using the paired $t$-test. This was done as previous work has shown that the paired t-test has a lower Type II error rate compared to non-parametric tests for Likert scale data [23]. It is therefore more likely to detect differences between the two techniques. A $p$-value of less than 0.05 indicated a significant difference.

\section{Results}

\section{Demographics and feasibility}

The mean age of population was $13.6 \pm 2.7$ years (median: 13.5, range: $7.0-18.3$ ) and $33(55 \%)$ were female. The mean heart rate was $82.3 \pm 15.9 \mathrm{bpm}$ (median: 82 , range: $50-114$ ). The full demographic information and patient diagnoses are shown in Table 2. Real-time and standard breath hold 
Table 2 Full demographic information and patient diagnoses

\begin{tabular}{ll}
\hline & $\begin{array}{l}\text { Mean } \pm \text { standard deviation } \\
\text { (range) }\end{array}$ \\
\hline Male/Female & $27 / 33$ \\
Age (years) & $13.6 \pm 2.7$ (7.0 to 18.3) \\
Height (m) & $1.6 \pm 0.2$ (1.2 to 1.9) \\
Weight (kg) & $47.5 \pm 15.8$ (23.1 to 82.0) \\
BSA & $1.4 \pm 0.3$ (0.9 to 2.0) \\
Heart rate (bpm) & $82 \pm 16$ (50 to 114) \\
Pulmonary Hypertension & 11 \\
Cardiomyopathy & 10 \\
Family history Sudden death/ & 9 \\
cardiomyopathy & \\
Pulmonary artery stenosis & 3 \\
Coarctation of the aorta & 4 \\
Tetralogy of Fallot (repaired) & 4 \\
Transposition of the great arteries (repaired) & 3 \\
Atrial septal defect & 3 \\
Ventricular Septal Defect & 2 \\
Aortopathy & 5 \\
Left ventricular outflow tract obstruction & 3 \\
Atrio-ventricular valve dysfunction & 3 \\
\hline BSA, body suffe area &
\end{tabular}

BSA, body surface area

imaging was successfully performed in all subjects. The total time acquisition time for the full breath hold stack was $348 \pm 79$ s (median: 335, range: 239-573) compared to $19.8 \pm 5.8 \mathrm{~s}$ for the acquisition of the real-time spiral stack (median: 19 s, range: $11-36$ ). The online CS reconstruction for each real-time slice (all phases) was $\sim 1.5 \mathrm{~s}$, with an additional $\sim 3.5 \mathrm{~s}$ per slice to send the data to the external computer and receive the reconstructed series back to the scanner database. In our buffered implementation, this resulted in all phases from all slices being available for viewing $\sim 28 \mathrm{~s}$ after the end of the acquisition.

\section{Image quality}

Representative images are shown in Figs. 2 and 3, and the corresponding movies can be seen in Additional files 1, 2, 3, and 4. These demonstrate that the spiral real-time images are of good quality. However, Fig. 4 shows examples of cases with residual aliasing artefacts and reduced edge definition (corresponding movies can be seen in Additional file 5 and Additional file 6), which is reflected in the qualitative image scores. Specifically, spiral real-time images had more residual aliasing artefacts than standard breath hold images (artefact score: $3.8 \pm 0.1$ vs $4.8 \pm 0.1, p<0.001$ ) and lower edge definition (edge score: $4.4 \pm 0.7$ vs $4.8 \pm 0.5, p<0.001)$. There was a trend towards poorer motion fidelity (motion score: $4.6 \pm 0.1$ vs $4.8 \pm 0.1, p=0.06)$. There were no significant

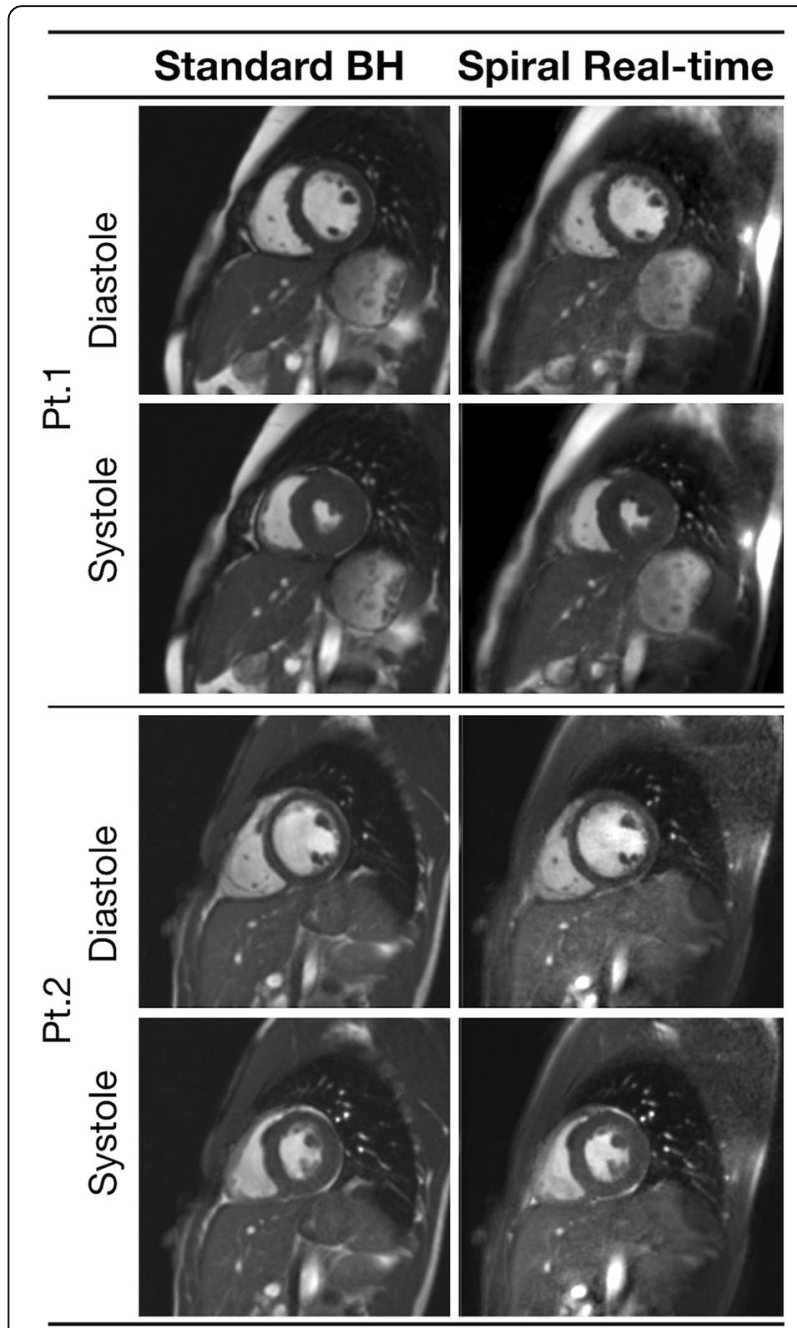

Fig. 2 Example image quality from two patients with left heart disease. Example image quality at systole and diastole in two patients with congenital left heart disease. Pt1: Left ventricular outflow tract obstruction, Pt2: Family history of cardiomyopathy (see Additional file 1 and Additional file 2 for corresponding movies); $\mathrm{BH}=$ breath hold

differences between qualitative image scores for the different observers $(p>0.1)$.

Quantitative edge sharpness was also significantly lower $(p<0.001)$ for the spiral real-time images compared to the standard $\mathrm{BH}$ images $\left(0.68 \pm 0.19 \mathrm{~mm}^{-1}\right.$ vs $0.53 \pm 0.16 \mathrm{~mm}^{-1}$, respectively). Additionally, quantitative estimates of image contrast (blood pool-to-myocardial signal intensity ratio) were significantly lower $(p<0.001)$ for the spiral real-time images compared to the standard $\mathrm{BH}$ images $(2.7 \pm 0.4$ vs. $3.2 \pm 0.4$, respectively).

\section{Ventricular volume quantification}

Ventricular metrics measured using spiral real-time and clinical standard breath hold imaging are shown in Table 3. Bland-Altman plots for LV metrics are shown in Figs. 5 and 6 . There was a small $(1.7 \mathrm{~mL})$ but significant 


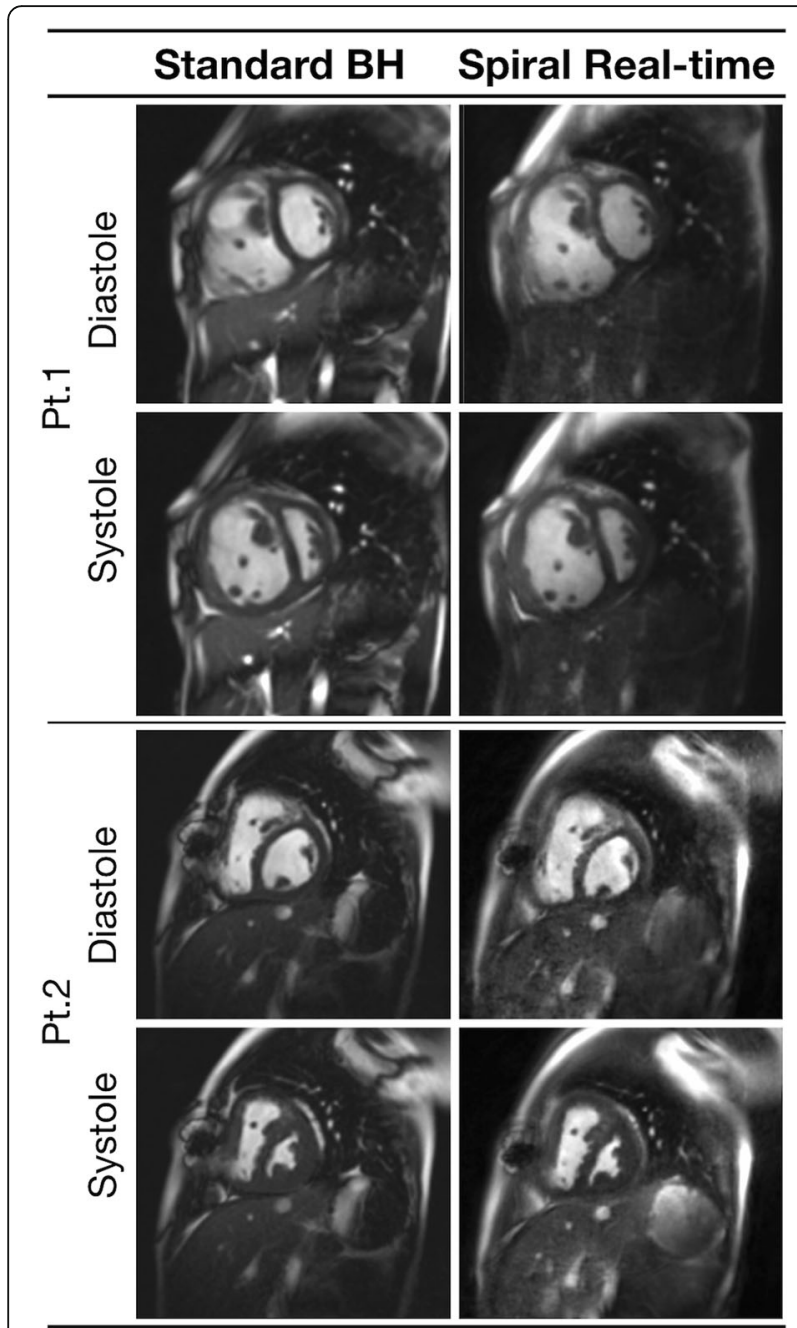

Fig. 3 Example image quality from two patients with right heart disease. Example image quality at systole and diastole in two patients with congenital left heart disease. Pt1: Idiopathic Pulmonary Hypertension, Pt2: Double outlet right ventricle (see Additional file 3 and Additional file 4 for corresponding movies)

underestimation of LV EDV, and a small $(1.0 \mathrm{~mL})$ but significant overestimation of LV ESV using the real-time imaging technique. This resulted in small underestimations of LV SV $(2.6 \mathrm{~mL})$ and LVEF (1.5\%). There was also a small but significant overestimation of LV mass $(3.1 \mathrm{~g})$. Nevertheless, the limits of agreement between real-time and breath hold data for all LV metrics were narrow. Body surface area correlated with the percentage difference between real-time and breath hold derived LV mass (rho $=-0.27, p=0.041)$. Differences for all other LV metrics were not associated $(p>0.05)$ with either heart rate or body surface area.

Bland-Altman plots for RV metrics are shown in Figs. 6 and 7. There were no significant differences in RV EDV or ESV, however there was a small underestimation of RV SV (1.8 mL) and RV EF (1.4\%). However,

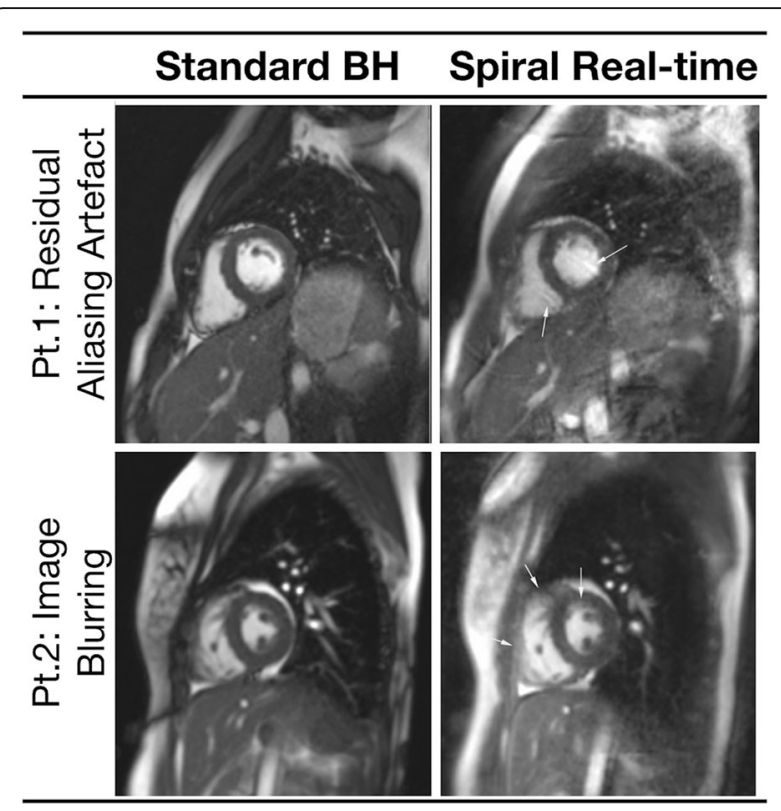

Fig. 4 Example image quality from two patients with imaging artefacts. Example image quality in two patients with residual imaging artefacts. Arrows highlight artefacts on spiral real-time images. Pt1: Shows residual aliasing artefact, Pt2: Shows image blurring (see Additional file 5 and Additional file 6. for corresponding movies)

the limits of agreement between real-time and $\mathrm{BH}$ data for all RV metrics were narrow. Differences in RV metrics were not significantly $(p>0.05)$ associated with either heart rate or body surface area.

The inter-observer CoV's and ICC's are shown in Table 4 and intra-observer CoV's and ICC's are shown in Table 5. The largely overlapping confidence intervals demonstrated that there were no significant differences in inter-observer and intra-observer variability between spiral real-time and standard breath hold derived RV and LV metrics.

\section{Discussion}

The main findings of this study were that: i) real-time bSSFP imaging using tiny golden-angle spiral trajectories combined with a CS reconstruction was feasible, ii) the image quality of the real-time technique was slightly lower than the standard breath hold technique, iii) there was good agreement for quantification of both LV and RV metrics between the spiral real-time and breath hold clinical standard techniques.

\section{Spiral bSSFP sequence with CS reconstruction}

Real-time CMR is particularly useful when imaging children, as it can be performed quickly and without breath holds. In this study, we implemented a novel real-time sequence that leveraged both efficient spiral $k$-space filling and CS reconstruction. Spiral $k$-space filling has previously 
Table 3 Primary observer; ventricular metrics measured using real-time and standard $\mathrm{BH}$ imaging

\begin{tabular}{|c|c|c|c|c|}
\hline & Real-time $^{a}$ & Standard Breath Hold ${ }^{a}$ & Bias & Limits of agreement \\
\hline LV EDV $(\mathrm{mL})$ & $110 \pm 38$ & $112 \pm 38^{b}$ & $1.7 \pm 4.6$ & -7.3 to 10.7 \\
\hline LV ESV (mL) & $40 \pm 16$ & $39 \pm 16^{b}$ & $-1.0 \pm 3.5$ & -7.9 to 5.9 \\
\hline LV SV (mL) & $70 \pm 25$ & $73 \pm 24^{b}$ & $2.6 \pm 4.8$ & -6.7 to 12.0 \\
\hline LV EF (\%) & $64 \pm 6$ & $66 \pm 7^{b}$ & $1.5 \pm 3.0$ & -4.3 to 7.3 \\
\hline LV mass & $88 \pm 36$ & $85 \pm 34^{b}$ & $-3.1 \pm 7.0$ & -16.9 to 10.6 \\
\hline RV EDV (mL) & $121 \pm 37$ & $121 \pm 38$ & $0.5 \pm 5.2$ & -9.6 to 10.6 \\
\hline RV ESV (mL) & $51 \pm 23$ & $49 \pm 24$ & $-1.2 \pm 5.0$ & -11.0 to 8.5 \\
\hline RV SV (mL) & $70 \pm 20$ & $72 \pm 20^{b}$ & $1.8 \pm 4.9$ & -7.8 to 11.4 \\
\hline RV EF (\%) & $59 \pm 9$ & $61 \pm 10^{b}$ & $1.4 \pm 3.7$ & -6.0 to 8.7 \\
\hline
\end{tabular}

$E D V$, end-diastolic volume; $E F$, ejection fraction; $E S V$, end-systolic volume, $L V$, left ventricular; $R V$, right ventricular; $S V$, stroke volume ${ }^{a}$ Displayed as mean \pm standard deviation

$\mathrm{b}$ Indicates significant differences with Standard BH technique $(p<0.05)$

Bias is the mean of the paired difference (Standard BH - Real-time) presented with standard deviation (SD) of the difference

Limits of agreements are bias $\pm 1.96 \times \mathrm{SD}$

been used to successfully accelerate spoiled gradient echo sequences (i.e. phase contrast CMR [24] and CMR angiography [25]). However, there are some problems associated with combining spiral trajectories with bSSFP readouts $[14,26,27]$. These mainly relate to off-resonance effects and longer repetitions times. Previous studies have shown that these effects can be partly mitigated using both zeroth and first order moment rewinders to balance the imaging gradients $[14,28]$. The problem with this approach is that it significantly increases repetition time and lowers temporal resolution. Therefore, we chose to use a shorter conventional zeroth order moment rewinder, combined with a relatively short readout $(1.86 \mathrm{~ms})$ to reduce off-resonance effects. However, this increases the number of interleaves required to fully sample $k$-space. Consequently, high acceleration factors were required to ensure adequate temporal resolution and we utilized CS to reconstruct artefact-free images. Our CS implementation shares many characteristics with the previously validated GRASP reconstruction for radial trajectories [18]. Specifically, we utilized tiny golden angle spacing to produce temporal incoherency and penalized temporal finite differences in the reconstruction. Combining spiral bSSFP with CS resulted in a real-time sequence with only slightly lower spatial resolution, and similar temporal resolution, to clinical standard breath hold imaging.
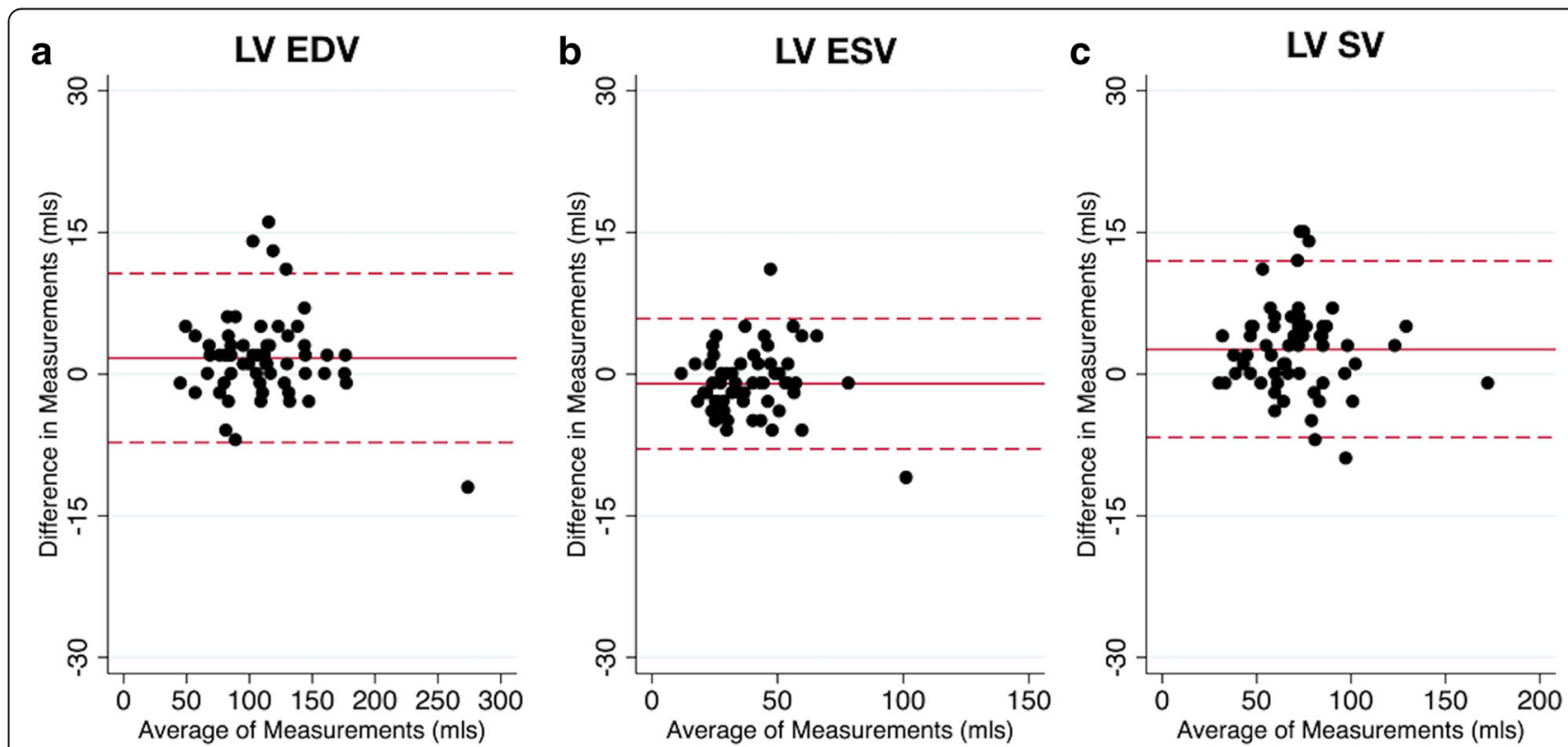

Fig. 5 Left ventricular (LV) Bland-Altman plots. Bland-Altman plots of standard breath-hold (BH) vs real-time techniques for left ventricular (a) enddiastolic volume (EDV), (b) end-systolic volume (ESV), (c) stroke volume (SV). The solid red line indicates the bias, with the dashed red lines showing the upper and lower limits of agreement (bias $\pm 1.96 \times$ StandardDeviation) between the two techniques 

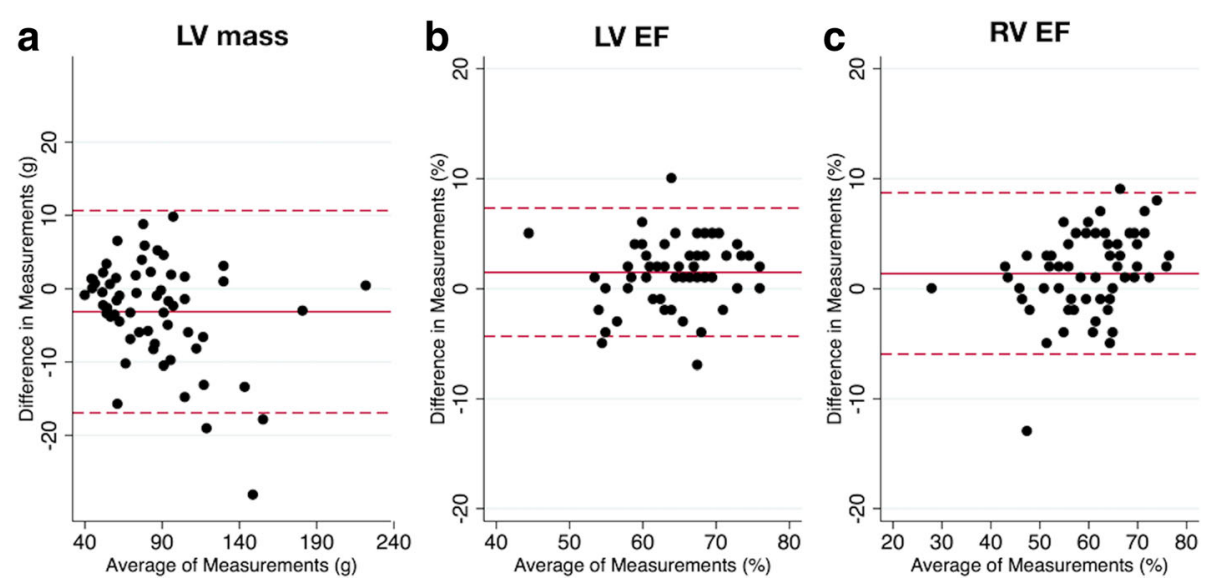

Fig. 6 Bland-Altman plots for LV ejection fraction (EF) and LV mass. Bland-Altman plots of standard BH vs real-time techniques for; (a) left ventricular mass, (b) left ventricular ejection fraction (EF), (c) right ventricular EF. The solid red line indicates the bias, with the dashed red lines showing the upper and lower limits of agreement (bias $\pm 1.96 \times$ StandardDeviation) between the two techniques

\section{Clinical utility}

Clinical utility depends on the ability of the reader to process the images to accurately measure ventricular volumes. In this study, images were processed by three independent CMR specialists (not involved in the development of the sequence) This is a more 'real-world' test of the sequence, in contrast to many studies in which a single observer (often involved in the development of the technology) is used.

We demonstrated that spiral real-time images were of diagnostic quality. However, they did have slightly lower edge definition and greater amount of residual aliasing artefact. This is not surprising as the real-time images had lower spatial resolution and were acquired with significant acceleration. Interestingly, although CS with temporal finite difference sparsity can result in temporal blurring, there was only a trend towards reduced motion fidelity.

Irrespective of differences in image quality, from a clinical point it is the ability to accurately measure ventricular volumes that is paramount. In this study, we found that LV EDV was smaller and LV ESV was bigger with real-time imaging compared to standard $\mathrm{BH}$ imaging. We also found similar differences in RV ESV, although this did not reach statistical significance. These biases were probably due to inaccurate segmentation of the real-time images, secondary to lower edge sharpness and myocardial blood pool contrast. In diastole, we believe these inaccuracies resulted in blood pool being included in the myocardial mass and EDV being underestimated. We believe the converse occurred in systole, with myocardial mass being included in the blood pool and ESV being overestimated.
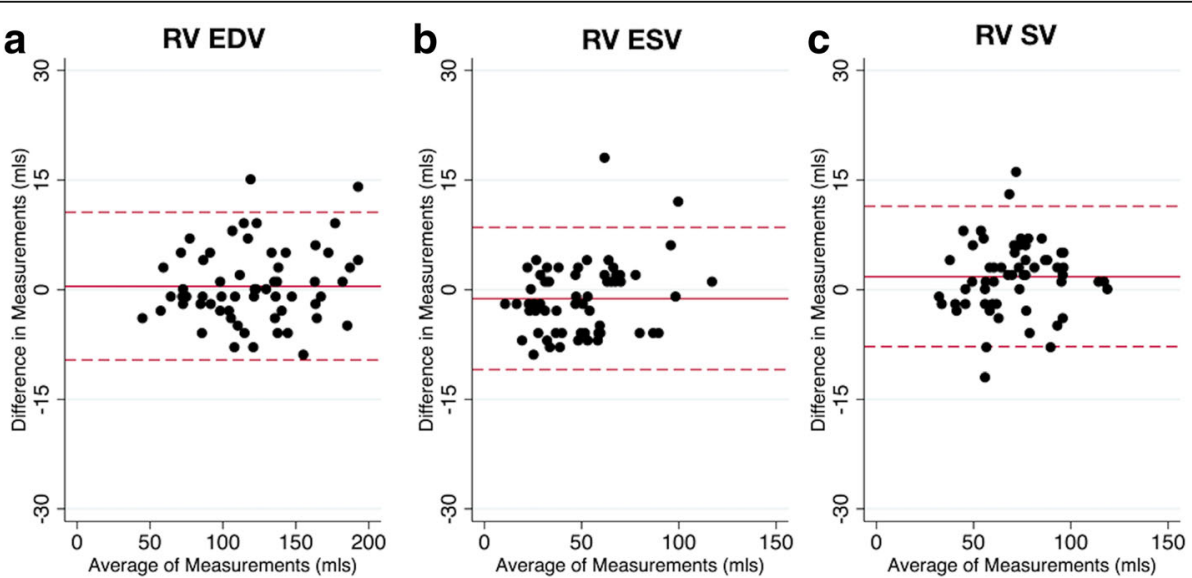

Fig. 7 Right ventricular Bland-Altman plots. Bland-Altman plots of standard breath hold vs real-time techniques for right ventricular; (a) EDV, (b) ESV, (c) SV. The solid red line indicates the bias, with the dashed red lines showing the upper and lower limits of agreement (bias $\pm 1.96 \times$ StandardDeviation) between the two techniques 
Table 4 Inter-observer variability; ventricular metrics measured using real-time and standard BH imaging

\begin{tabular}{|c|c|c|c|c|}
\hline & Standard Breath Hold CoV ${ }^{a}$ & Real-time CoV ${ }^{\mathrm{a}}$ & Standard Breath Hold ICC & Real-time ICC \\
\hline LV EDV (\%) & 4.1 (3.1 to 5.2) & $4.0(2.9$ to 5.0$)$ & 0.983 (0.964 to 0.992) & 0.984 (0.968 to 0.992$)$ \\
\hline LV ESV (\%) & 7.0 (5.2 to 8.9$)$ & 8.7 (6.4 to 11.0$)$ & 0.973 (0.946 to 0.987 ) & 0.931 (0.862 to 0.967 ) \\
\hline LV SV (\%) & 7.1 (5.3 to 9.0 ) & 6.8 (5.1 to 8.6$)$ & 0.958 (0.914 to 0.980$)$ & 0.952 (0.902 to 0.977 ) \\
\hline LV EF (\%) & $4.2(3.1$ to 5.3$)$ & 5.7 (4.2 to 7.2$)$ & 0.891 (0.785 to 0.946$)$ & 0.772 (0.578 to 0.884 ) \\
\hline RV EDV (\%) & 6.6 (4.9 to 8.3 ) & 6.9 (5.1 to 8.8$)$ & 0.964 (0.926 to 0.983 ) & 0.956 (0.911 to 0.979$)$ \\
\hline RV ESV (\%) & 13.2 (9.7 to 16.8$)$ & 12.8 (9.4 to 16.3$)$ & 0.968 (0.935 to 0.985) & 0.954 (0.906 to 0.978 ) \\
\hline RV SV (\%) & 10.9 (8.0 to 13.9$)$ & 11.5 (8.5 to 14.6$)$ & 0.865 (0.738 to 0.933 ) & 0.835 (0.684 to 0.917 ) \\
\hline RV EF (\%) & 7.1 (5.3 to 9.0$)$ & 7.7 (5.7 to 9.7 ) & 0.836 (0.687 to 0.918 ) & 0.785 (0.599 to 0.891 ) \\
\hline
\end{tabular}

${ }^{a}$ Displayed as mean (95\% confidence intervals)

An important question in children, is whether the differences between real-time and standard breath hold imaging were associated with heart rate or body size. In this study, the only significant association we found was between percentage overestimation of LV mass and decreasing BSA. This was probably due to errors in epicardial segmentation in smaller children, possibly due to factors such as less pericardial fat and geometric considerations. Nevertheless, the biases were all below $5 \%$ and would be expected to have minimal effect on clinical decision making. More importantly, the limits of agreement were narrow compared to previously described real-time methods (including those that utilize CS) $[6,29]$. We believe that this is due to the high spatial and temporal resolution of our spiral real-time sequence, which was only slightly lower than the standard breath hold sequence. In this study, we also examined inter-observer and intra-observer variability (half the populations tested for each). For the standard breath hold sequence the coefficients of variation and ICC's were similar to previously published data [30], with RV data having greater variability than LV data. This is to be expected due to the greater difficulty in segmenting the more trabeculated and complex shaped RV. Importantly, the inter-observer and intra-observer variability of real-time sequences were similar to standard breath hold imaging. This is an important finding, as demonstrating reliability is vital for clinical translation. We believe that our findings show that spiral real-time imaging can successfully be used for assessment of paediatric heart disease. The main benefit over standard breath hold imaging is that it can be acquired during free breathing. Another important secondary benefit is that a whole ventricular stack can be acquired in approximately $20 \mathrm{~s}$, as opposed to almost $6 \mathrm{~min}$ for standard breath hold imaging. Thus, this technique holds the potential to significantly shorten CMR scan times in children.

\section{Limitations}

The main limitation of this study was the minimum age in the population was 7 years, which limits the translatability of our findings to younger children and infants. The reason for this age limit was that younger children and infants would have found it difficult to perform the standard breath hold sequence necessary for validation. Nevertheless, future studies concentrating on optimization and validation of this technique for infants would be useful. Another limitation was that we did not assess RV mass in this study. Several studies have suggested that RV mass may be an important predictive marker in certain diseases [31]. However, RV mass is difficult to accurately quantify in patients without RV hypertrophy, even when using standard breath hold imaging. Therefore, we did not include it this study, although it could be included in future studies concentrating on patient with RV disease. It should also be noted that the real-time imaging was

Table 5 Intra-observer variability; ventricular metrics measured using real-time and standard breath hold imaging

\begin{tabular}{|c|c|c|c|c|}
\hline & Standard Breath Hold CoV ${ }^{a}$ & Real-time $\mathrm{CoV}^{\mathrm{a}}$ & Standard Breath Hold ICC & Real-time ICC ${ }^{a}$ \\
\hline LV EDV (\%) & $1.7(1.3$ to 2.1$)$ & 1.5 (1.1 to 1.9$)$ & 0.994 (0.987 to 0.997) & 0.997 (0.994 to 0.999$)$ \\
\hline LV ESV (\%) & 4.3 (3.2 to 5.5 ) & 4.7 (3.5 to 6.0 ) & 0.985 (0.968 to 0.993 ) & 0.984 (0.967 to 0.992 ) \\
\hline LV SV (\%) & 2.3 (1.7 to 2.9$)$ & 2.6 (1.9 to 3.2$)$ & 0.993 (0.985 to 0.997) & 0.994 (0.988 to 0.997 ) \\
\hline LV EF (\%) & 1.8 (1.4 to 2.3 ) & 2.4 (1.8 to 3.0$)$ & 0.955 (0.909 to 0.978 ) & 0.919 (0.839 to 0.961$)$ \\
\hline RV EDV (\%) & 2.0 (1.5 to 2.5$)$ & 2.2 (1.6 to 2.7$)$ & 0.994 (0.987 to 0.997) & 0.994 (0.987 to 0.997) \\
\hline RV ESV (\%) & 4.2 (3.1 to 5.3 ) & 5.0 (3.7 to 6.3$)$ & 0.989 (0.977 to 0.995 ) & 0.977 (0.952 to 0.989 ) \\
\hline RV SV (\%) & 3.6 (2.6 to 4.5 ) & 4.2 (3.1 to 5.3 ) & 0.989 (0.977 to 0.995 ) & 0.985 (0.968 to 0.993 ) \\
\hline RV EF (\%) & 2.7 (2.0 to 3.4 ) & 3.4 (2.5 to 4.3 ) & 0.975 (0.949 to 0.988 ) & 0.946 (0.890 to 0.974$)$ \\
\hline
\end{tabular}

${ }^{\mathrm{a}}$ Displayed as mean (95\% confidence intervals) 
performed during free breathing and physiological variation may account for some discrepancies with standard breath hold imaging. However, the differences probably remain small because the slices in the real-time short axis stack are all acquired at in different points in the respiratory cycle. Thus, any discrepancies may 'average out' when summed across the whole ventricle. In terms of intra-observer variability, there was no prescribed time delay between processing of data, as volumes (including repeated volumes) were presented data in a randomized manner. However, both the standard breath hold and real-time data was treated in the same manner and the variability measures were in keeping with previously published data. Thus, we believe the similar intra-observer variability of the real-time and standard breath hold data does reflect the clinical reality. Finally, one disadvantage of compressed sensing is long reconstruction times. In this study, we used an online GPU based reconstruction that fed images back to the scanner in a clinically acceptable time frame $(<30 \mathrm{~s}$ for the entire short axis stack). Nevertheless, more work is required so that reconstruction times can be reduced to that of standard Cartesian imaging.

\section{Conclusion}

In conclusion, we implemented a novel spiral real-time bSSFP sequence with CS reconstruction. We showed good agreement between biventricular volumes measured using spiral real-time and standard breath hold techniques in children with heart disease. Thus, we believe that this technique could be used successfully in the paediatric population.

\section{Additional files}

Additional file 1: Movie 1: Cine showing image quality in one patient with left heart disease: Left ventricular outflow tract obstruction. (MOV $2013 \mathrm{~kb}$ )

Additional file 2: Movie 2: Cine showing image quality in one patient with left heart disease: Family history of Cardiomyopathy. (MOV $3451 \mathrm{~kb}$ )

Additional file 3: Movie 3: Cine showing image quality in one patient with right heart disease: Idiopathic Pulmonary Hypertension. (MOV 3015 kb)

Additional file 4: Movie 4: Cine showing image quality in one patient with right heart disease: Double outlet right ventricle. (MOV $1885 \mathrm{~kb}$ )

Additional file 5: Movie 5: Cine showing residual aliasing artfeact in the spiral real-time images in one patient. (MOV $2454 \mathrm{~kb}$ )

Additional file 6: Movie 6: Cine showing residual blurring in the spiral real-time images in one patient. (MOV $1990 \mathrm{~kb}$ )

\section{Abbreviations}

BH: Breath-hold; BSA: Body surface area; bSSFP: Balanced steady state free precession; CMR: Cardiovascular magnetic resonance; CNR: Contrast-to-noise ratio; CoV: Coefficient of variation; CS: Compressed sensing; EDV: End-diastolic volume; EF: Ejection fraction; ESV: End-systolic volume; GRASP: Golden-angle Radial Sparse Parallel; ICC: Intra-class correlation; LV: Left ventricle/left ventricular; RV: Right ventricle/right ventricular; SD: Standard deviation; SNR: Signal-to noise ratio; SV: Stroke volume; tGA: Tiny Golden Angle; TR: Repetition time

\section{Acknowledgements}

We would like to express our gratitude to our clinical and research CMR radiographers in Great Ormond Street Hospital, London. This work was supported by the National Institute for Health Research Biomedical Research Centre at Great Ormond Street Hospital for Children National Health Service Foundation Trust and University College London.

\section{Funding}

JAS receives Royal Society-EPSRC funding; Dorothy Hodgkin Fellowship (DH130079). GK receives Heart Research UK funding.

\section{Availability of data and materials}

The datasets used and analyzed during the current study are available from the corresponding author on reasonable request.

\section{Authors' contributions}

JAS and VM performed study design and were major contributors in writing the manuscript. JAS developed spiral bSSFP sequence, OsiriX plugins and performed Edge sharpness measurements. VM performed statistics and developed Python script for qualitative image scoring. GK developed online Compressed Sensing reconstruction algorithm. OT, MH and KM analyzed patient data in terms of ventricular volumes and qualitative image scoring. All authors read and approved the final manuscript.

\section{Ethics approval and consent to participate}

The local committee of the UK National Research Ethics Service approved the study (06/Q0508/124), and written consent was obtained from all subjects/guardians.

\section{Consent for publication}

Permissions: All parents of participants and participants gave consent and assent to participate in the study. Permission was also obtained from parents to publish anonymized patient data collected.

\section{Competing interests}

The authors declare that they have no competing interests.

\section{Publisher's Note}

Springer Nature remains neutral with regard to jurisdictional claims in published maps and institutional affiliations.

\section{Author details}

${ }^{1}$ UCL Centre for Cardiovascular Imaging, Institute of Cardiovascular Science, University College London, 30 Guildford Street, London WC1N 1EH, UK. ${ }^{2}$ Cardiorespiratory Unit, Great Ormond Street Hospital for Children, London WC1N 3JH, UK.

Received: 30 April 2018 Accepted: 23 October 2018

Published online: 06 December 2018

References

1. Grothues F, Smith GC, Moon JCC, Bellenger NG, Collins P, Klein HU, Pennell DJ. Comparison of interstudy reproducibility of cardiovascular magnetic resonance with two-dimensional echocardiography in normal subjects and in patients with heart failure or left ventricular hypertrophy. Am J Cardiol. 2002;90:29-34.

2. Oosterhof T, van Straten A, Vliegen HW, Meijboom FJ, van Dijk APJ, Spijkerboer AM, Bouma BJ, Zwinderman AH, Hazekamp MG, de Roos A, Mulder BJM. Preoperative thresholds for pulmonary valve replacement in patients with corrected tetralogy of Fallot using cardiovascular magnetic resonance. Circulation. 2007;116:545-51.

3. Muthurangu V, Lurz P, Critchely J, Deanfield J, Taylor A, Hansen MS. Realtime assessment of right and left ventricular volumes and function in patients with congenital heart disease by using high spatiotemporal resolution radial k-t SENSE. Radiology. 2008;248:782-91.

4. Lustig M, Donoho D, Pauly JM. Sparse MRI: the application of compressed sensing for rapid MR imaging. Magn Reson Med. 2007;58:1182-95.

5. Chan RW, Ramsay EA, Cheung EY, Plewes DB. The influence of radial undersampling schemes on compressed sensing reconstruction in breast MRI. Magn Reson Med. 2012;67:363-77. 
6. Haji-Valizadeh H, Rahsepar AA, Collins JD, Bassett E, Isakova T, Block T, Adluru G, DiBella EVR, Lee DC, Carr JC, Kim D. Validation of highly accelerated real-time cardiac cine MRI with radial $k$-space sampling and compressed sensing in patients at 1.5T and 3T. Magn Reson Med. 2018;79: 2745-51.

7. Kido T, Kido T, Nakamura M, Watanabe K, Schmidt M, Forman C, Mochizuk $T$. Compressed sensing real-time cine cardiovascular magnetic resonance: accurate assessment of left ventricular function in a single-breath-hold. J Cardiovasc Magn Reson. 2016;18:50

8. Feng L, Srichai MB, Lim RP, Harrison A, King W, Adluru G, Dibella EVR, Sodickson DK, Otazo R, Kim D. Highly accelerated real-time cardiac cine MRI using k-t SPARSE-SENSE. Magn Reson Med. 2013;70:64-74.

9. Voit D, Zhang S, Unterberg-Buchwald C, Sohns JM, Lotz J, Frahm J. Realtime cardiovascular magnetic resonance at $1.5 \mathrm{~T}$ using balanced SSFP and 40 ms resolution. J Cardiovasc Magn Reson. 2013;15:79.

10. Kowalik G, Courot A, Steeden J, Muthurangu V. Golden-angle spiral sparse parallel phase-contrast MR acquisition with an on-line fast GPU based reconstruction for high resolution real-time cardiovascular assessments. In Proc Intl Soc Mag Reson Med 25; Honolulu, Hawaii. 2017:1262. http:// archive.ismrm.org/2017/1262.html.

11. Winkelmann S, Schaeffter T, Koehler T, Eggers H, Doessel O. An optimal radial profile order based on the Golden ratio for time-resolved MRI. IEEE Trans Med Imaging. 2007;26:68-76.

12. Lingala SG, Zhu Y, Kim Y-C, Toutios A, Narayanan S, Nayak KS. A fast and flexible MRI system for the study of dynamic vocal tract shaping. Magn Reson Med. 2017;77:112-25.

13. Hargreaves BA, Nishimura DG, Conolly SM. Time-optimal multidimensional gradient waveform design for rapid imaging. Magn Reson Med. 2004;51:81-92.

14. Nayak KS, Hargreaves BA, Hu BS, Nishimura DG, Pauly JM, Meyer CH. Spiral balanced steady-state free precession cardiac imaging. Magn Reson Med. 2005;53:1468-73.

15. Wundrak S, Paul J, Ulrici J, Hell E, Rasche V. A small surrogate for the Golden angle in time-resolved radial MRI based on generalized Fibonacci sequences. IEEE Trans Med Imaging. 2015;34:1262-9.

16. Kowalik GT, Steeden JA, Pandya B, Odille F, Atkinson D, Taylor A, Muthurangu V. Real-time flow with fast GPU reconstruction for continuous assessment of cardiac output. J Magn Reson Imaging. 2012;36:1477-82.

17. Kowalik GT, Steeden JA, Muthurangu V. Implementation of a generalized heterogeneous image reconstruction system for clinical magnetic resonance. Concurrency and Computation: Practice and Experience. 2015;27:1603-11.

18. Feng L, Grimm R, Block KT, Chandarana H, Kim S, Xu J, Axel L, Sodickson DK, Otazo R. Golden-angle radial sparse parallel MRI: combination of compressed sensing, parallel imaging, and golden-angle radial sampling for fast and flexible dynamic volumetric MRI. Magn Reson Med. 2014;72:707-17.

19. McKenzie CA, Yeh EN, Ohliger MA, Price MD, Sodickson DK. Self-calibrating parallel imaging with automatic coil sensitivity extraction. Magn Reson Med. 2002:47:529-38.

20. Rosset A, Spadola L, Ratib O. OsiriX: an open-source software for navigating in multidimensional DICOM images. J Digit Imaging. 2004;17:205-16.

21. Steeden JA, Atkinson D, Hansen MS, Taylor AM, Muthurangu V. Rapid flow assessment of congenital heart disease with high-spatiotemporal-resolution gated spiral phase-contrast MR imaging. Radiology. 2011;260:79-87.

22. Bland JM, Altman DG. Statistics notes: measurement error proportional to the mean. BMJ. 1996;313:106.

23. Meek GE, Ozgur C, Dunning K. Comparison of the t vs. Wilcoxon signedrank test for Likert scale data and small samples. J Mod Appl Stat Methods. 2007;6:10.

24. Kowalik GT, Knight DS, Steeden JA, Tann O, Odille F, Atkinson D, Taylor A, Muthurangu V. Assessment of cardiac time intervals using high temporal resolution real-time spiral phase contrast with UNFOLDed-SENSE. Magn Reson Med. 2015;73:749-56.

25. Steeden JA, Pandya B, Tann O, Muthurangu V. Free breathing contrastenhanced time-resolved magnetic resonance angiography in pediatric and adult congenital heart disease. J Cardiovasc Magn Reson. 2015;17:38.

26. Narayan G, Nayak K, Pauly J, Hu B. Single-breathhold, four-dimensional, quantitative assessment of LV and RV function using triggered, real-time, steady-state free precession MRI in heart failure patients. J Magn Reson Imaging. 2005;22:59-66.
27. Barkauskas KJ, Rajiah P, Ashwath R, Hamilton Jl, Chen Y, Ma D, Wright KL, Gulani V, Griswold MA, Seiberlich N. Quantification of left ventricular functional parameter values using 3D spiral bSSFP and through-time nonCartesian GRAPPA. J Cardiovasc Magn Reson. 2014;16:65.

28. Feng X, Salerno M, Kramer CM, Meyer CH. Non-Cartesian balanced steadystate free precession pulse sequences for real-time cardiac MRI. Magn Reson Med. 2016;75:1546-55.

29. Kido T, Kido T, Nakamura M, Watanabe K, Schmidt M, Forman C, Mochizuki T. Assessment of left ventricular function and mass on free-breathing compressed sensing real-time cine imaging. Circ J. 2017;81:1463-8.

30. Marchesseau S, Ho JX, Totman JJ. Influence of the short-axis cine acquisition protocol on the cardiac function evaluation: a reproducibility study. Eur J Radiol Open. 2016;3:60-6.

31. Valente AM, Gauvreau K, Assenza GE, Babu-Narayan SV, Schreier J, Gatzoulis MA, Groenink M, Inuzuka R, Kilner PJ, Koyak Z, et al. Contemporary predictors of death and sustained ventricular tachycardia in patients with repaired tetralogy of Fallot enrolled in the INDICATOR cohort. Heart. 2014;100:247-53.

\section{Ready to submit your research? Choose BMC and benefit from:}

- fast, convenient online submission

- thorough peer review by experienced researchers in your field

- rapid publication on acceptance

- support for research data, including large and complex data types

- gold Open Access which fosters wider collaboration and increased citations

- maximum visibility for your research: over $100 \mathrm{M}$ website views per year

At BMC, research is always in progress.

Learn more biomedcentral.com/submissions 